\title{
Antimicrobial Resistance Profile of Clinically Isolated Bacteria at Wolaita Sodo University Teaching Referral Hospital
}

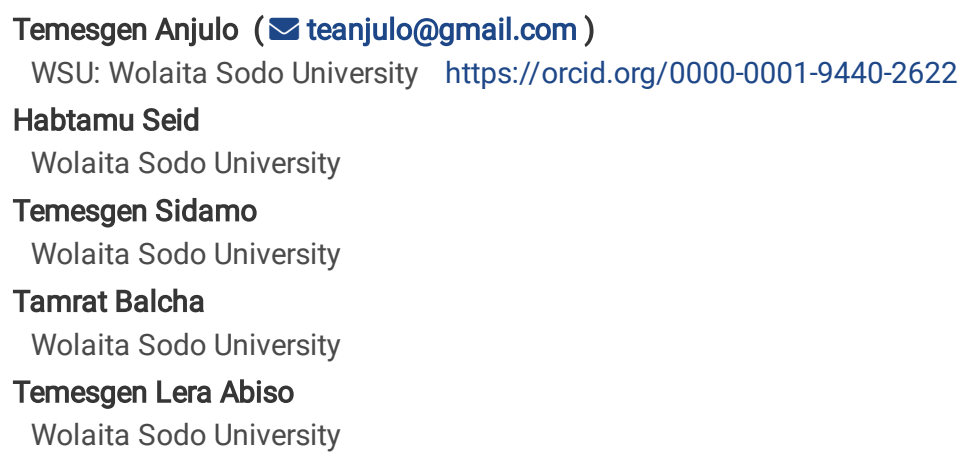

\section{Research Article}

Keywords: Resistance, Bacteria, Antibiotic, gram positive, gram negative, isolates.

Posted Date: July 7th, 2021

DOI: https://doi.org/10.21203/rs.3.rs-645376/v1

License: (c) (i) This work is licensed under a Creative Commons Attribution 4.0 International License. Read Full License 


\section{Abstract}

Background: Antibiotic resistance is a serious threat to the human populations everywhere. However, less attention is given to its concern in subSaharan Africa including Ethiopia. There is information gap for the prescribers regarding antibiotic resistance and its pattern. The aim of this study was to review the antimicrobial resistance pattern of bacteria in Wolaita Sodo University Teaching Referral Hospital.

Methods: Three years retrospective data of cultures and records of 330 patients were used to analyze the antibiotic sensitivity pattern. The statistical softwares including Epidata 3.5.1 and SPSS version 21 were used. Laboratory records lacking age, sex, culture isolation or drug susceptibility test data were excluded.

Results: Out of 330 samples extracted from the microbiology laboratory, 150 samples were culture positive for bacteria isolates. From positive culture growth, $73.3 \%$ were gram positive bacteria whereas $26.7 \%$ were gram negative. Staphylococcus aureus was the most prevalent isolate among gram-positive isolates as Escherichia coli was for gram negative isolates. Overall antimicrobial resistance of gram positive isolates was $54.2 \%$ where as that of gram negative bacteria was $60.0 \%$.

Conclusion: As per this study, S. aureus and E. coli were the pathogenic isolates of highest prevalence among gram positive and gram-negative bacteria, respectively, in the study population. Most of the isolated pathogens showed high resistance towards the commonly prescribed antimicrobial agents.

\section{Introduction}

Antibiotic resistance (AMR) is the ability of microorganisms to resist antimicrobials. It is a major public health problem threatening a range of infectious microorganisms [1]. Although it may happen naturally, misuse of antibiotics in humans and animals is the leading cause of antimicrobial resistance [2]. Resistance to antibiotics occurs through modified antimicrobial target, enzymatic hydrolysis, changes in cell membrane response and impermeability. Multi-antibiotic resistant bacteria acquire resistance by mutation and gene transfer via conjunctions, transformation or transduction[3].

AMR makes the antibiotics ineffective letting the pathogenic bacteria continue in the body [2][3]. The rise in resistance not only impedes our ability to treat infections but also has broader societal and economic impacts. Therefore, it endangers the achievement of the Sustainable Development Goals. $[5,6]$ The expenditure of health care for patients with resistant infections is higher than that for non-resistant infections because of longer duration of illness, additional tests and the need for more expensive medicines [4]. The consequences of AMR are aggravated in situations such as civil unrest, violence, famine and natural disasters as well as in settings with poor health care services [5, 7]. Indeed, AMR is a major public health concern in both developed and developing countries and it can affect people without discriminating against age, sex, race or country [4, 8]. However, most of its impacts fall on low- and middle-income countries pertaining to lack of infrastructure, human and financial resources to adequately counter drug resistance epidemics [6]. The other reasons are high prevalence of infectious diseases, lack of trained health professionals, irrational use of drugs and limited set-up of microbiological laboratory [4, 8]. According to WHO report of 2014, in five out of six WHO regions, Escherichia Coli resistance to third generation Cephalosporins and fluoroquinolones and that of Staphylococcus aureus to methicillin was higher than 50\%. The report claimed $45 \%$ of deaths in both Africa and South-East Asia were because of multi-antibiotic resistant bacteria. It further revealed that third generation cephalosporin resistant K. pneumonia caused high death incidences in Africa (77\%), Eastern Mediterranean region (50\%), South East Asia (81\%) and Western Pacific region (72\%) [8]. Moreover, death report attributable to AMR bacteria of Asia in 2016 was 4,730,000 and that of Africa was $4,150,000$ [9].

The aim of this study was to review the antimicrobial resistance pattern of bacteria isolated from different retrospectively inoculated specimens at Wolaita Sodo University Teaching Referral Hospital.

\section{Methods}

\subsection{Study Setting and Design}

The study was conducted at Wolaita Sodo University Teaching Referral Hospital (WSUTRH) which is located in Wolaita Zone, $154 \mathrm{~km}$ from Hawassa, capital of South nations, nationalities and peoples region, and $329 \mathrm{~km}$ from Addis Ababa, capital of Ethiopia. The hospital provides general outpatient and inpatient services including medical, surgical, pediatric, psychiatric, ophthalmic, gynecology and Obstetrics Emergency care. Annual patient volume is around 200,000 with its 370 beds.

\subsubsection{Study Design and Period:}

The institution based three-year (2016-2018) retrospective study design was used for the period from 2016-2018. Data was collected from June 20/2019-July 20/2019.

\subsubsection{Source Population}


Data of all patients who had attended Wolaita Sodo University Teaching Referral Hospital, South Ethiopia during 2016-2018 were included.

\subsubsection{Study Population:}

The records of patients who requested culture and gave a sample for microbiological data during 2016-2018 were included.

\subsubsection{Inclusion and Exclusion Criteria}

All completed patient data with full information registered in the logbook during the study period were included and patient data with incomplete data were excluded.

\subsubsection{Data Collection:}

Demographic data of patients, profiles of the bacteria isolated including their antimicrobial susceptibility were retrieved from Wolaita Sodo teaching referral University hospital (WSUTRH) microbiology laboratory unit registration records using a standard data collection form. Laboratory records which had incomplete information of age, sex, culture or drug susceptibility test results were excluded.

The Microbiology laboratory receives specimens like urine, stool, body fluid, blood, pus and swab from different discharges. The sample received from different wards, Emergency patients, intensive care units and outpatients services inoculated on different culture media based on SOP (Standard operating Procedures), growth was observed after 16-24hrs incubation at $37 \mathrm{C}^{0}$. In case of blood stream infection was suspected, blood culture media trypticase Soy broth was prepared in the laboratory and blood sample inoculated aseptically. If there was hemolysis, gas and turbidity observed, it was subcultured on appropriate media for further isolation. MacConkey and Blood agar Media were used to isolate non fastidious bacteria and Chocolate agar media was used to culture fastidious bacteria. If the Sample was collected from genital area Thayer Martin agar media was used. If growth was observed, single colony from culture selected and gram staining was performed. Based on their gram reaction, biochemical tests selected. Gram negative bacteria were further identified using oxidase, citrate utilization test, urease, lysine iron agar, mannitol fermentation, indole test. Gram positive bacteria were identified using catalase, coagulase, bacitracin, optochin, bile solubility and Novabicin.

Some bacteria were identified by species level using biochemical and some of the identified by genus level by gram stain reaction (by their colony characteristics and morphology). Antibiotic sensitivity pattern such as susceptibility, intermediate and resistance were conducted with Kirby-Bauer disc diffusion method and interpretation was based on clinical and laboratory standard Institute (CLSI) 2014-2017 guideline. Different antibiotic discs (Abtek LTD, UK) were used: Ampicillin (AMP) $(10 \mu \mathrm{g})$, gentamicin-Gen(10 $\mu \mathrm{g})$, ciprofloxacin-CRP(5 $\mu \mathrm{g})$, ceftriaxone-CRT(30 $\mu \mathrm{g})$, ceftazidimeCAZ(30 $\mu \mathrm{g})$, norfloxacin-NOR) $(10 \mu \mathrm{g})$, nitrofurantoin-NIT(300 $\mu \mathrm{g})$, augmentin-AUG(20/10 $\mu \mathrm{g})$, cotrimoxazole-COT(1.25/23.75 $\mu \mathrm{g})$, chloramphenicolCAF $(30 \mu \mathrm{g})$, meropenem-MER $(10 \mu \mathrm{g})$, tetracycline-TAT $(30 \mu \mathrm{g})$, penicillin G-PEN(10 IU), Clindamycin-CLD $(2 \mu \mathrm{g})$ and erythromycin-ERY $(15 \mu \mathrm{g})$ and etc.

\section{Bacteria antimicrobial pattern result interpretation}

\section{Susceptible (S)}

The 'susceptible' category implies that isolates are inhibited by the usual achievable concentration of antimicrobial agent when the recommended dosage is used for the site of infection.

\section{Intermediate (I)}

The 'intermediate' category includes isolates with antimicrobial agent MICs that approach usually attainable blood and tissue levels and for which response rates may be lower that for susceptible isolates. The intermediate category implies clinical efficacy in body sites where the drugs are physiologically concentrated or when a higher than normal dosage of a drug can be used. This category also includes a buffer zone, which should prevent small, uncontrolled, technical factors from causing major discrepancies in interpretation, especially for drugs with narrow pharmacotoxisity margin.

\section{Resistant (R)}

The 'resistant' category implies that isolates are not inhibited by the usually achievable concentrations of the agent with normal dosage schedules, and/or that demonstrate MICs or zone diameters that fall in the range where specific microbial resistant mechanisms are likely, and clinical efficacy of the agent against the isolate has not been reliably shown in treatment studies

\section{Quality Assurance:}

A standard bacteriological procedure was followed to maintain correct laboratory test results before testing all the inoculated samples. American Type Culture collection (ATCC) standard reference strains of Escherichia coli (E. coli) ATCC-25922, Staphylococcus aureus (S. aureus) ATCC 25923 and Pseudomonas aeruginosa (P. aeruginosa) ATCC-25853 were used to control the quality of the culture and drug susceptibility testing. All data were checked for consistency and completeness.

\section{Ethical Considerations}


The ethical clearance was obtained from the ethical review committee of Wolaita Sodo University, College of Health Science and medicine. Formal consent was also obtained from Wolaita Sodo University Referral Hospital. For privacy reason, all data was kept confidential. Anonymity of records was maintained by using registration number and unique code numbers used by service providers at Wolaita Sodo University teaching referral hospital.

\section{Data Processing and Analysis}

The preliminary data were checked and processed depending on the study variables. Data analysis was done by using SPSS Version-20 software. The results were summarized using means and percentages and presented by using graphs and tables.

\section{Results}

\section{Socio-demographic Characteristics of the Subjects}

From data records of a total of 330 patients over the years 2016 through 2018, those which fulfill the inclusion criteria were extracted. Out of 330,167 (50.6\%) were males and 163 were females (49.4\%). The majority of the age groups, 87 (26.45) were from 25 to 34 and the least group included 38 (11.5\%) in this study were in the age 46 years and above. From total 330 patients, 124(37.6\%) visited the microbiological laboratory in the year 2017 (See Table 1).

\section{Bacterial growth status and sample inoculated at WSUTRH, 2016-2018}

The age group from which $37(24.7 \%)$ the highest bacteria growth observed was 15-24 years, and the least 17 (11.7\%) was 35-44years ('Table 2'). Out of the total specimens inoculated, 150 (45.5\%) presented positive bacterial growth whereas 180 (54.5\%) didn't show any growth. In terms of the specimen types used for the bacterial isolation, 159 (48.2\%) were discharges from vagina, urethra and wound; 49 (14.8\%) were from stool; 71 (21.5\%) were from urine and 51 (15.5\%) were from other body fluids ('See table 3').

\section{Types of Bacteria Isolated from Positive Growth}

The top three bacteria isolated from those positive growths in this study were S. aureus 92 (61.3\%), E.coli 19 (12.7\%) and S. saprophyte 9 (6\%). (See Table 4). Among those 150 positive growths, based on their gram reaction, 110 (73.3\%) were gram positive and 40(26.7\%) were gram negative. Gram positive bacteria identified were $S$. auras, S. pyogen, $S$, pneumonia, and S. saprophytes where as gram negative bacteria isolated include E.coli, $P$. aurogenus, Protous Spp, Salmonella Spp, shigella Spp and Nisserai Spp ('See figure 1').

\section{Antimicrobial Resistance Profile among Gram Positive Bacteria Isolated}

Gram positive bacteria identified were $S$. auras, S. saprophytes, S. pyogen and S. pneumonia. The overall antimicrobial resistances for $S$. aureus, $S$. saprophytes, S. pyogen, and S. pneumonia were rated as 53\%, 62\%, 74 and 52\%, respectively. With respect to specific antibiotics, resistance against S. aureus was $88 \%$ for ampicillin, $66 \%$ for gentamicin, $71 \%$ for chloramphenicol, $61 \%$ for ceftriaxone, $69 \%$ for Nalidxic acid, $80 \%$ for amoxicillin, $67 \%$ for tetracycline and $82 \%$ for vancomycine ('Table 5 ').

\section{Antimicrobials Resistance among Gram Negative Bacteria}

The gram negative bacteria identified were E. coli, P. aurogenous, Proteus Spp, ShigellaSpp, Salmonella Spp and Nisseria Spp. Overall resistance for all antimicrobial resistance of E. coli was 52\%, P. aurogenous 75\%, proteus Spp 65\%, shigella Spp 66\%, Salmonella 56\% and Nisseria Spp 63\% ('See table $\left.6^{\prime}\right)$.

\section{Discussion}

In the present study, antimicrobial resistance of gram positive isolates was $54.2 \%$ and that of gram negative bacteria was $60.0 \%$ whereas the overall antimicrobial resistance in this study was $57.1 \%$. S. auras was $92(61.3 \%)$ the most prevalent isolate from all bacteria growth and E.coli $19(12.7 \%)$ was the second prevalent isolate. Antibiotic resistance of S. auras, S. saprophytes, S. pyogenes, S. pneumonia, E. coli, P. aurogenous, Proteus Spp, Nisseria Spp and Salmonella Spp were 53\%.53\%,62\%,74\%, 52\%, 52\%, 75\%, 65\%, 63\%, respectively. The antimicrobial resistance in the present study was lower than that conducted in Debere Markos Referral Hospital, Ethiopia, $84.6 \%$ for gram positive and $72.2 \%$ for gram negative bacteria and southern Ethiopia University Teaching Hospitals 74.6\% for gram positive and $84.0 \%$ for gram negative bacteria [14-16]. This could be due to some updates in microbiological set-ups and knowledge of prescriber in choices of drugs or better awareness of prescribers [13]. On the other hand, as per this study, the resistance reported is higher than that reported by the studies conducted in the Africa countries which revealed antimicrobial resistance of $34.6 \%$ in Benin, 31.9\% in Congo, 14.3\% in Togo and 16.3\%in Madagascar [10].

In the present study, Staphylococcus was predominant and E. coli was the next prevalent one which indicated that the results are similar to the study conducted in Gabon, central Africa[4]. Overall resistance of E.coli in this study was $52 \%$ and in the meta-analysis conducted in Ethiopia the 
antimicrobial resistance of E.coli was 45.38\% (33.5\%-57.7\%) the highest resistance in Addis Ababa and the lower resistance found in the Tigray Region $27.5 \%$ [17]. Resistance of S.auras for all antimicrobial tastings was $53 \%$ which is lower than study conducted in Greek which was $88 \%$ [18].

The lower resistance finding in this study compared to that of the study conducted in Greek was that in this study the sample isolate was from skin infection where there's increased population of normal flora.

Another study conducted in Rewand Referral Hospital, of 241Gram-negative isolates tested for ceftriaxone, 183 (75.9\%) were resistant[19]. The finding of the present study is similar to the above finding.

\section{Strength and Limitation of the Study}

For the nature of the retrospective study, detailed socio-demographic information and clinical status of patients was not incorporated. The number of drugs tested on some pathogens was small in number.

\section{Conclusion And Recommendation}

As per this study, $S$. auras and $E$. coli are the pathogenic isolates of highest prevalence among gram positive and gram negative bacteria, respectively, in the study population. Most of the isolated pathogens showed high resistance against ampicillin, gentamicin, chloramphenicol, ceftriaxone, Nalidxic acid, amoxicillin, tetracycline and vancomycin. It needs collaboration among clinicians, laboratory personnel and pharmacy professionals against antimicrobial resistance and using culture and antimicrobial sensitivity testing.

\section{Abbreviations (Acronyms And Abbreviations)}

AMR, antimicrobial resistance; ATCC, American Type Culture collection; MDR, multidrug resistance; SNNPR, South nations nationalities and peoples region; Spp, Species; WHO, world health organizations; WSUTRH,Wolaita Sodo University Teaching Referral Hospital.

\section{Declarations}

\section{Acknowledgment}

We would like to forward our gratitude to Wolaita Sodo University, College of Health Sciences and Medicine. We also thank Data Collectors.

\section{Authors' contribution}

TA, HS, TS, TB and TL

These authors equally contributed to this research work

\section{Funding}

This study was not funded

\section{Availability of Data \& Materials}

The data for this research is available, so we can contact you when you need our data for the future process.

\section{Ethics approval and consent to participate}

The ethical clearance was obtained from the Ethical review committee of Wolaita Sodo University, College of Health Science and medicine. Formal permission was also obtained from Wolaita Sodo University Referral Hospital. For reasons of privacy, all data were kept confidential. Anonymity of records was maintained by using registration number and unique code numbers used by service providers at Wolaita Sodo University teaching referral hospital.

\section{Consent for publication}

Not applicable

\section{Competing interests}

The authors have declared that no competing interests exist.

\section{References}

1. WHO. WHO Report on Surveillance of Antibiotic Consumption 2016-2018 Early implementation. Report, 2018. 
2. Commission E. Staff Working Paper of the Services of the Commission on

AntimicrobialResistance.Availableonline:http/ec.europa.eu/food/food/biosafety/salmonells/ antimicrobial resistance. Food and Biosafety, 2014.

3. Moges F, Mulu EM, Tessema A, Belyhun B, Shiferaw Y. Y, et al., The growing challenges of antibacterial drug resistance in Ethiopia.. 2014. doi:10.1016/j.jgar.2014.02.004. JGAR, 2014.

4. Alabi AS, et al. Retrospective analysis of antimicrobial resistanceand bacterial spectrum of infection in Gabon, Central Africa. BMC; 2013.

5. WHO., Antimicrobial resistance (WHO Fact sheet). Geneva: World Health Organization; February 2018 (http://www.who.int/en/newsroom/factsheets/detail/antimicrobial-resistance,accessed 25 September 2018). WHO fact sheet, 2018.

6. Bank W. The World Bank. Drug resistant infections: a threat to our economic future. Washington D.C:TheWorldBank;2017. Final reoprt; 2018. (http://documents.worldbank.

7. Founou RC, Essack FL. SY, Clinical and economic impact of antibiotic resistance in developing countries: $A$ systematic review and metaanalysis. PLoS ONE 12(12, 2017.

8. WHO. ANTIMICROBIAL RESISTANCE Global Report on Surveillance Report, 2014.

9. UNGA. AMR was the 4th health agenda that followed a Political Declaration. 2016.

10. Peggy S, Lai LMB, Meney C, Valeri L, Michelle C, White. Epidemiology of antibiotic-resistant wound infections from six countries in Africa. BMJ Glob Health; 2018.

11. WangaiFK M. LuleGN,Karari EM,MaritimMC,JaokoWG,etal, Bridging antimicrobial resistance knowledge gaps:The East African perspective on a global problem. PLoSONE, 2019.

12. Seboxa T, Abebe AW, Tsegaye W, Hailu AAT W, et al., High Mortality from Blood Stream Infection in Addis Ababa, Ethiopia, Is Due to Antimicrobial Resistance. PLoS ONE 2015: p. 10.

13. Institute EpH, Ethiopia Antimicrobial Resistance Surveillance. surveillance, 2018.

14. Wondemagegn Mulu BA, Yimer M, Hailu T, Ayele H, Abate D. Bacterial agents and antibiotic resistance profles of infections from diferent sites that occurred among patients at Debre Markos Referral Hospital, Ethiopia: a cross-sectional study. BMC, 2017.

15. al Ae. The burden of antimicrobial resistance at tertiary care hospital, southern Ethiopia: a three years' retrospective study. BMC Infectious Diseases; 2019.

16. Solomon FB, F.W.W., Amsalu Amache Arota and Yishak Leka Abraham, Antibiotic resistant airborne bacteria and their multidrug resistance pattern at University teaching referral Hospital in South Ethiopia. Ann Clin Microbiol Antimicrob (2017) 16:29 2017.

17. Kald B, Tuem AKG. Tesfay Mehari Atey ,Helen Bitew, and a.D.F.B. Ebrahim M. Yimer, Drug Resistance Patterns of Escherichia coli in Ethiopia: A Meta-Analysis. BioMed Research International, 2018: p. 13.

18. Christina Stefanak Al, Matoula T, CC,Evaggelia Polythodoraki, Chryssou S-E, George Kontochristopoulos and Christina Antoniou, Six-Year Retrospective Review of Hospital Data on Antimicrobial Resistance Profile of Staphylococcus aureus Isolated from Skin Infections from a Single Institution in Greece. antibiotics 2017.

19. SutherlandT MC, NziyomazeE NiyomugaboJ-P, Niyonsenga Z, MuvunyiCM, et al. (2019)Widespread antimicrobial resistanceamongbacterialinfectionsin a Rwandan referralhospital.PLoSONE 14(8):e0221121.https://doi.org/10.1371/journal.pone.0221121.

\section{Tables}

Table1. Socio-demographic characteristics of patients involved in Antimicrobial resistance profiling at WSUTRHL, 2016-2018. 


\begin{tabular}{|c|c|c|c|}
\hline Variable $(\mathrm{N}=330)$ & & Number & Percentage (\%) \\
\hline \multirow[t]{2}{*}{ Sex } & Male & 167 & 50.6 \\
\hline & Female & 163 & 49.4 \\
\hline \multirow[t]{6}{*}{ Age group } & $<5$ years & 55 & 16.7 \\
\hline & $5-14$ & 38 & 11.5 \\
\hline & $15-24$ & 66 & 20.0 \\
\hline & $25-34$ & 87 & 26.4 \\
\hline & $35-44$ & 46 & 13.9 \\
\hline & 44years and above & 38 & 11.5 \\
\hline \multirow[t]{3}{*}{ Years patient visited microbiology lab } & 2016 & 117 & 35.5 \\
\hline & 2017 & 124 & 37.6 \\
\hline & 2018 & 89 & 27.0 \\
\hline
\end{tabular}

Table 2: Age distribution of patients from which the bacterial isolates obtained at WSUTRHL, 2016-2018.

\begin{tabular}{|llllllll|}
\hline Growth status & \multicolumn{2}{l}{ Patient age group } & & & & \multicolumn{2}{c|}{ Total } \\
\cline { 2 - 7 }$(\mathrm{N}=330)$ & $<5$ years & $5-14 y e a r s$ & 15-24years & 25-34years & 35-44years & 45years and above & 150(100\%) \\
Positive culture & $26(17.3 \%)$ & $19(12.7 \%)$ & $37(24.7 \%)$ & $30(20.0 \%)$ & $17(11.3 \%)$ & $21(14.0 \%)$ & $180(100)$ \\
\hline Negative & $29(16.1 \%)$ & $19(10.6 \%)$ & $29(16.1 \%)$ & $57(31.7 \%)$ & $29(16.1 \%)$ & $17(9.4 \%)$ & $330(100 \%)$ \\
\hline Total & $55(16.7 \%)$ & $38(11.5 \%)$ & $66(20.0 \%)$ & $87(26.4 \%)$ & $46(13.9 \%)$ & $38(11.5 \%)$ & \\
\hline
\end{tabular}

Table 3: Distribution Bacterial growth status and sample inoculated at WSUTRH, 20162018.

\begin{tabular}{|c|c|c|c|}
\hline Variable $(\mathrm{N}=330)$ & & Number & Percentage $(\%)$ \\
\hline \multirow[t]{2}{*}{ Growth status } & Positive & 150 & 45.5 \\
\hline & Negative & 180 & 54.5 \\
\hline \multirow[t]{4}{*}{ Types of Specimens used for bacterial isolation } & Urine & 71 & 21.5 \\
\hline & *Discharges and swab & 159 & 48.2 \\
\hline & Body fluid & 51 & 15.5 \\
\hline & Stool & 49 & 14.8 \\
\hline
\end{tabular}

Table 4: Prevalent of pathogenic bacteria among positive isolation at WSUTRH, 2016-2018 


\begin{tabular}{|lll|}
\hline Variable $(\mathrm{n}=150)$ & Frequency & Percentage (\%) \\
\hline S. aureus* & 92 & 61.3 \\
\hline E.coli & 19 & 12.7 \\
\hline S. saprophytes* & 9 & 6.0 \\
\hline P. aurogenous & 8 & 5.3 \\
\hline S. pneumonia* & 5 & 3.3 \\
\hline S. pyogenes* & 4 & 2.7 \\
\hline Proteus Spp. & 4 & 2.7 \\
\hline Shigella & 4 & 2.7 \\
\hline Neisseria Spp. & 3 & 2.0 \\
\hline Salmonella & 2 & 1.3 \\
\hline Total & 150 & $100 \%$ \\
\hline
\end{tabular}

Table 5: Antimicrobial resistance among gram positive isolates at WSUTRH, 2016-2018

\begin{tabular}{|c|c|c|c|c|c|c|c|c|c|}
\hline \multirow[t]{2}{*}{ antibiotics assayed } & \multicolumn{2}{|l|}{ S.auras } & \multicolumn{2}{|c|}{ S. saprophytes } & \multicolumn{2}{|c|}{ S. pyogenes } & \multicolumn{2}{|c|}{ S. pneumonia } & \multirow{2}{*}{$\begin{array}{l}\text { Total } \\
\text { R }\end{array}$} \\
\hline & $\mathrm{S}(\%)$ & $\mathrm{R}(\%)$ & $\mathrm{S}(\%)$ & $\mathrm{R}(\%)$ & $\mathrm{S}(\%)$ & $\mathrm{R}(\%)$ & S (\%) & $\mathrm{R}(\%)$ & \\
\hline Ampicillin & $4(12)$ & $29(88)$ & NA & NA & 0 & $2(100)$ & $1(100)$ & 0 & 31 \\
\hline Gentamicin & 18(34) & $35(66)$ & $1(25)$ & $3(75)$ & 0 & $2(100)$ & 0 & $3(100)$ & 43 \\
\hline Cloxaciline & $20(48)$ & $22(52)$ & $2(33)$ & $4(67)$ & 0 & $1(100)$ & $1(50$ & $1(50)$ & 28 \\
\hline Chlorampencol & 16(29) & $40(71)$ & 0 & $4(100)$ & 0 & $2(100)$ & 0 & $2(100)$ & 48 \\
\hline Ceftrazone & 28(39) & $44(61)$ & 0 & $9(100)$ & 0 & $3(100)$ & $2(40)$ & $3(60)$ & 59 \\
\hline Nalidexin & $5(31)$ & 11(69) & $2(40)$ & $3(60)$ & $1(100)$ & 0 & $1(50)$ & $1(50)$ & 15 \\
\hline Ciprofloxacilin & $70(86)$ & 11(14) & $5(55)$ & $4(45)$ & $2(75)$ & $1(25)$ & $5(100)$ & 0 & 16 \\
\hline Clindamycine & $45(67)$ & $22(33)$ & $6(75)$ & $2(25)$ & $1(50)$ & $1(50)$ & $2(50)$ & $2(50)$ & 27 \\
\hline Cotrimoxazole & $5(45)$ & $6(55)$ & 0 & $2(100)$ & 0 & 1(100) & $1(50)$ & $1(50)$ & 10 \\
\hline Amoxicillin & $2(20)$ & $8(80)$ & $1(50)$ & $1(50)$ & NA & NA & NA & NA & 9 \\
\hline Erythromycin & $22(55)$ & $18(45)$ & $1(25)$ & $3(75)$ & $1(50)$ & $1(50)$ & $1(100)$ & 0 & 22 \\
\hline Tetracycline & $4(33)$ & $8(67)$ & 0 & $1(100)$ & NA & NA & 0 & 1 & 10 \\
\hline Augumentin & 14(43) & 18(57) & $2(66)$ & $1(34)$ & NA & NA & NA & NA & 19 \\
\hline Cephalexine & 11(46) & 13(54) & $2(66)$ & $1(34)$ & NA & NA & 1 & 1 & 15 \\
\hline Vancomicine & $3(18)$ & 14(82) & $1(100)$ & 0 & NA & NA & 0 & 1 & 15 \\
\hline Total S \&R & $267(47 \%)$ & $299(53 \%)$ & $23(38)$ & $38(62)$ & $5(26)$ & $14(74)$ & $15(48)$ & $16(52)$ & $367(54.2 \%)$ \\
\hline
\end{tabular}

** S- susceptible, R- resistance, NA- not applicable

Table 6: Antimicrobial resistance among gram negative isolates at WSUTRH, 2016-2018. 


\begin{tabular}{|c|c|c|c|c|c|c|c|c|c|c|c|c|c|}
\hline \multirow{2}{*}{$\begin{array}{l}\text { Antibiotics } \\
\text { assayed for } \\
\text { sensitivity }\end{array}$} & \multicolumn{2}{|l|}{ E.coli } & \multicolumn{2}{|c|}{ P. aurogenous } & \multicolumn{2}{|c|}{ Proteus Spp. } & \multicolumn{2}{|c|}{ ShigellaSpp } & \multicolumn{2}{|c|}{ Salmonella } & \multicolumn{2}{|c|}{ Neisseria Spp. } & \multirow[t]{2}{*}{ Total R\% } \\
\hline & S (\%) & $\mathrm{R}^{\star}(\%)$ & S (\%) & $\mathrm{R}^{\star}(\%)$ & $\mathrm{S}(\%)$ & $\mathrm{R}^{*}$ & $\mathrm{~S}$ & $\mathrm{R}^{*}$ & S & $\mathrm{R}^{\star}$ & $\mathrm{S}$ & $\mathrm{R}^{*}$ & \\
\hline Ampicillin & 0 & $5(100)$ & 0 & 2 & 1 & 2 & 0 & 2 & 0 & 1 & 0 & 1 & 13 \\
\hline Gentamicin & 4 & $7(63.6)$ & 1 & 2 & 3 & 1 & 1 & 1 & 1 & 1 & 2 & 1 & 13 \\
\hline Cloxaciline & 2 & $3(60)$ & 0 & 1 & 0 & 1 & 0 & 1 & 0 & 0 & 0 & 0 & 6 \\
\hline Chlorampencol & 7 & $8(53)$ & 1 & 1 & 2 & 2 & 1 & 1 & 0 & 1 & 2 & 0 & 13 \\
\hline Ceftrazone & 5 & $9(64)$ & 2 & 6 & 1 & 2 & 3 & 1 & 0 & 0 & 0 & 2 & 20 \\
\hline Nalidexin & 7 & $1(15)$ & 1 & 3 & 1 & 1 & 0 & 1 & 1 & 0 & 0 & 0 & 6 \\
\hline Ciprofloxacilin & 12 & $4(25)$ & 2 & 6 & 2 & 2 & 2 & 2 & 0 & 0 & 1 & 2 & 16 \\
\hline Clindamycine & 3 & $7(70)$ & 1 & 6 & 0 & 2 & 0 & 2 & 0 & 1 & 1 & 0 & 18 \\
\hline Cotrimoxazole & 1 & $6(86)$ & 0 & 0 & 0 & 0 & 1 & 1 & 0 & 0 & 0 & 1 & 8 \\
\hline Amoxicillin & 0 & $3(100)$ & 1 & 0 & 0 & 1 & 0 & 0 & 0 & 0 & 0 & 0 & 4 \\
\hline Erythromycin & 5 & $0(0)$ & 1 & 4 & 0 & 1 & 0 & 0 & 1 & 0 & 0 & 1 & 6 \\
\hline Tetracycline & 1 & $2(70)$ & 0 & 1 & 0 & 2 & 0 & 2 & 0 & 1 & 0 & 1 & 9 \\
\hline Augumentin & 4 & $3(42)$ & 1 & 0 & 0 & 1 & 0 & 0 & 0 & 0 & 0 & 1 & 5 \\
\hline Cephalexine & 4 & $1(20)$ & 0 & 1 & 0 & 0 & 0 & 1 & 1 & 0 & 0 & 0 & 3 \\
\hline Vancomicine & 1 & $1(50)$ & 0 & 0 & 0 & 0 & 0 & 0 & 0 & 0 & 0 & 0 & 1 \\
\hline Total S \&R & $56(48)$ & $60(52)$ & $11(25)$ & $33(75)$ & $10(35)$ & $18(65)$ & $8(34)$ & $15(66)$ & $4(44)$ & $5(56)$ & $6(37)$ & $10(63)$ & $141(60 \%)$ \\
\hline
\end{tabular}

** S- susceptible, R- resistance, NA- not applicable

Figures

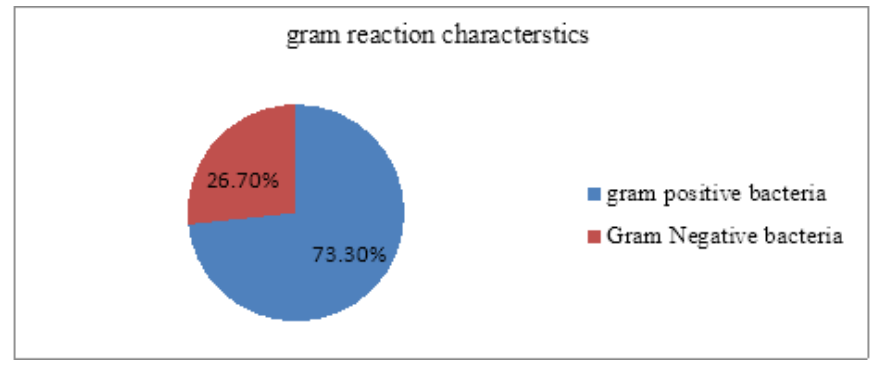

Figure 1

Isolated pathogenic bacteria based on their gram reaction characteristics at WSUTRH, 2016-2018 